\title{
Nonlinear Condition Tolerancing Using Monte Carlo Simulation
}

\author{
JOUILEL Naima \\ High School of engineering ENSAM Meknes \\ Research Laboratory in Mechanical, Mechatronic \& Control \\ - L2MC. Morocco \\ ELGADARI M'hammed \\ High School of engineering ENSAM Meknes \\ Research Laboratory in Mechanical, Mechatronic \& Control \\ - L2MC. Morocco
}

\author{
RADOUANI Mohammed \\ High School of engineering ENSAM Meknes \\ Research Laboratory in Mechanical, Mechatronic \& Control \\ - L2MC. Morocco \\ EL FAHIME Benaissa \\ High School of engineering ENSAM Meknes \\ Research Laboratory in Mechanical, Mechatronic \& Control \\ - L2MC. Morocco
}

\begin{abstract}
To ensure accuracy and performance of the products, designers tend to hug the tolerances. While, manufacturers prefer to increase them in order to reduce costs and ensure competition. The analysis and synthesis of tolerances aim on studying their influence on conformity with functional requirements. This study may be conducted in the case of the most unfavorable configurations with the "worst case" method, or "in all cases" using the statistical approach. However, having a nonlinear condition make it difficult to analyse the influence of parameters on the functional condition.

In this work, we are interested in the tolerance analysis of a mechanism presenting a nonlinear functional condition (slider crank mechanism). To do this we'll develop an approach of tolerances analysis combining the worst case and the statistical methods.
\end{abstract}

Keywords-Worst case tolerancing; statistical tolerancing; Monte Carlo simulation; nonlinear condition; slider crank system

\section{INTRODUCTION}

The industrial challenges lead to develop models and design support tools to meet customer needs by optimizing the triptych: cost quality and time. Several works have been conducted in order to analyze the impact of deviating component surfaces on functions, properties and assemblability of the product $[1,2,3]$.

Mechanical products tolerancing consist on specifying limits of dimensional characteristics variations (often unidirectional) with a tolerance interval. There are mainly two tolerancing approaches:

- "worst case", ensures the assembly and functionality of the mechanical system, but leads to a higher production comparing to the second approach;

- "statistic", ensures a low cost of production but accepts mechanisms whose functionality is not respected.

The tolerance analysis aims to study the influence of dimensional characteristics variations on the respect of functional requirements. This analysis may be conducted in the case of the most unfavorable configurations "worst case" or "in all cases" using the statistical approach. The analysis is so difficult to be performed in the case of the nonlinear functional conditions, because we cannot write them as an algebraic combination [4]. Our work aims to propose an approach based on the combining of the worst case and the statistical methods that allows the tolerance analysis and synthesis in the case of a nonlinear functional conditions.

This paper is organized as follows: In section 2 we treat tolerance analysis at worst case and we present its limits on tolerancing nonlinear condition. Section 3 describes the statistical tolerancing method using Monte Carlo Simulation. In section 4, we apply our approach on a slider crank system that presenting a nonlinear functional condition.

\section{TOLERANCE ANALYSIS "AT Worst CASE"}

The analysis at "Worst Case" (WC) is mainly used to operate the system in the most adverse conditions $[5,6,7]$. It allows finding extreme values (maximum and minimum) that can be reached by the resulting operating condition for any combination of the real initial dimensions.

In the case of mono-domain systems, the relationship between the resulting functional requirement and basic characteristics is often translated into a linear additive relationship. Overall, it resulted in a unidirectional relationship as follows:

$Y=\sum_{\substack{k=1 \\ \text { Where: }}}^{n} \alpha_{k} X_{k}=\sum_{k=1}^{n} \alpha_{k}\left(X_{k n} \pm \frac{t_{k}}{2}\right)$

- $\mathrm{Y}$ is the functional condition which must be between $\mathrm{y}_{\min }$ and $\mathrm{y}_{\max }$;

- $X_{\mathrm{kn}}$ is the nominal value of $X_{k}$;

- $\alpha_{k}$ is the influence coefficient $\left(a_{k}= \pm 1\right)$;

- $\mathrm{t}_{\mathrm{k}}$ is tolerance.

In this case, the functional condition often reaches its limit values only for a linear combination of limit values (minimum and / or maximum) of partial components dimensions such as: 


$$
\begin{gathered}
y_{\min } \leq\left(\sum_{k=1}^{n} \alpha_{k} X_{k}\right)_{\min }=\sum_{k=1}^{n} \alpha_{k} X_{k n}-\frac{1}{2} \sum_{k=1}^{n}\left|\alpha_{k}\right| t_{k} \\
\left(\sum_{k=1}^{n} \alpha_{k} X_{k}\right)_{\text {max }}=\sum_{\substack{k=1 \\
\leq}} \alpha_{k} X_{\text {max }}+\frac{1}{2} \sum_{k=1}^{n}\left|\alpha_{k}\right| t_{k}
\end{gathered}
$$

The functional condition is then satisfied if:

$$
y_{\text {max }}-y_{\text {min }}=t_{y} \leq \sum_{k=1}^{n}\left|\alpha_{k}\right| t_{k}
$$

Practically, the relationship between the resulting functional requirement and basic characteristics is more complex than the linear additive relationship.

\section{TOLERANCES ANALYSIS WITH MONTE CARLO SIMULATION}

Monte Carlo simulation (MCS) is a method for predicting errors manufacturing [8]. Its core idea is to use random samples of parameters or inputs to explore the behavior of a complex system or process. For this, the Monte Carlo simulation uses pseudo-random generators with numbers corresponding to different types of statistical distributions. By using Monte Carlo Simulation, the results obtained are more realistic than those obtained by conventional methods of calculation.

The user must define the random distribution of input variables. The number of experiments generated must be large enough to reliably determine the statistical parameters of output variables.

The simulation defines a statistical data generally described by the mean dimension:

$\mu=\frac{1}{N} \sum_{i=1}^{N} Z_{i}$

And standard deviation:

$$
\sigma=\sqrt{\frac{1}{N} \sum_{i=1}^{N}\left(Z_{i}-\mu\right)^{2}}
$$

Where:

- $\mathrm{Z}_{\mathrm{i}}$ is the value of the resulting dimension to the ' $\mathrm{i}_{\mathrm{th}}$ ', simulation cycle;

- $\mathrm{N}$ is the total number of simulation cycles;

The general approach of applying the MCS method is presented in analyzing functional condition tolerances is presented in Figure 1.

For the evaluation of the realization frequency of the functional condition, the statistical group is converted into a histogram (Figure 2).

The standard deviation ' $\sigma$ ' is a parameter characterizing the dispersion or variation of the values distribution around an average. Higher are the values concentrated around the average, lower is the standard deviation. In a normal distribution, the standard deviation ' $\sigma$ ' is used to establish confidence intervals for desired confidence levels. The production process is often considered satisfactory at $\pm 3 \sigma$. So 99.73\% of assemblies are in the interval $\left[\mu_{\mathrm{J}}-3 \sigma_{\mathrm{J}} ; \mu_{\mathrm{J}}+3 \sigma_{\mathrm{J}}\right]$ (Figure 3). For centric distribution, the functional requirement will be respected for $99.73 \%$ of assemblies if:

Tolerance on the requirement $=6 \sigma$

Modeling: choosing a feature that represents the model

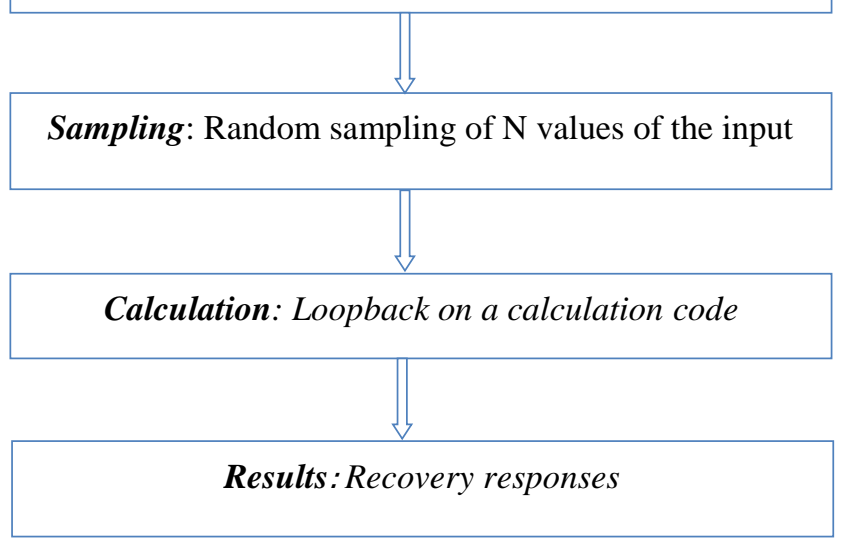

Fig. 1. General approach of applying the MCS

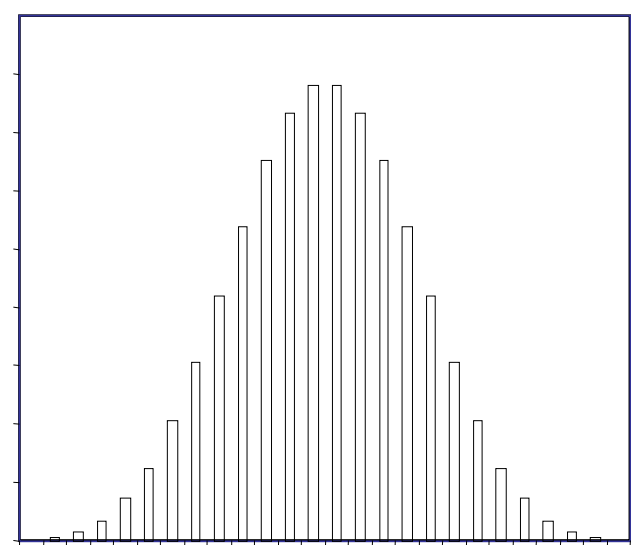

Fig. 2. View as histogram

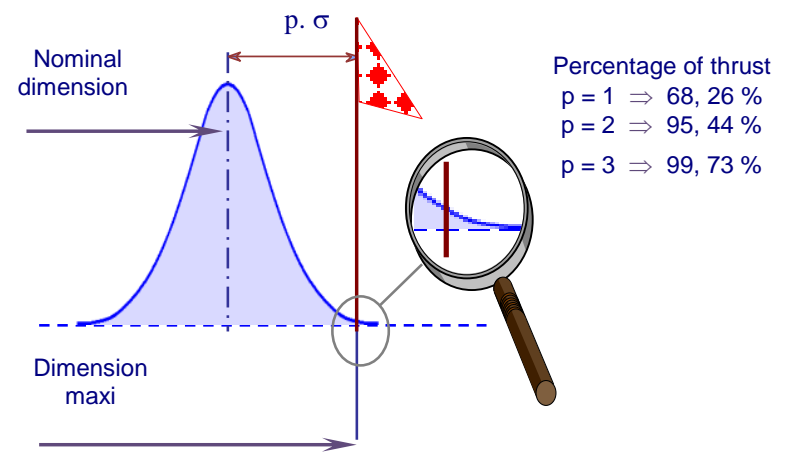

Fig. 3. Percentage of trust in an interval

\section{APPROACH OF TOLERANCING}

To solve the problem of the functional condition nonlinearity,the following approach is adopted based on both: 
Worst Case tolerancing and Monte Carlo Simulation. This combination will ensure system's functionality with large

tolerance intervals (figure 4).

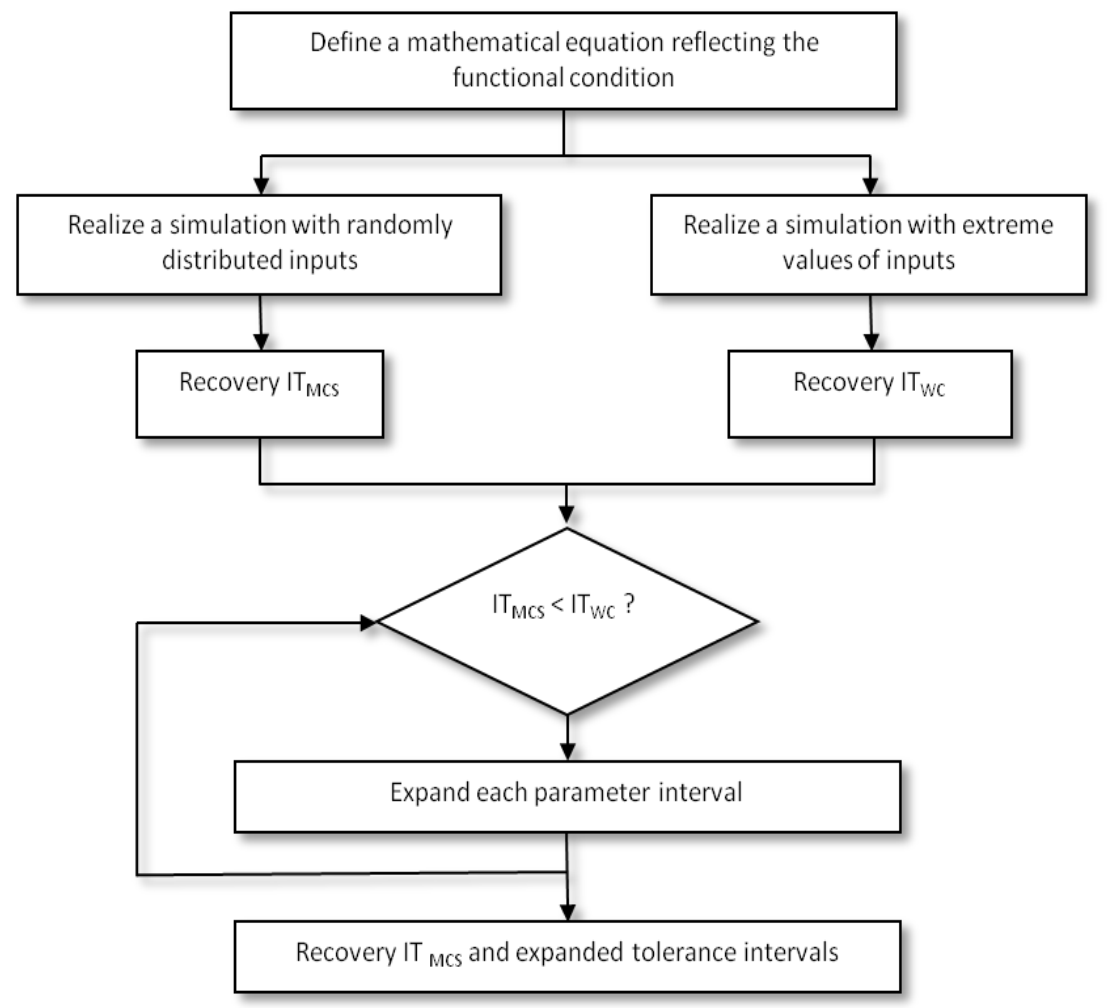

Fig. 4. flowchart of analyzing nonlinear condition tolerance

\section{CASE of Study}

A. System Overview

The case of slider crank system (Figure 4), the position $\mathrm{X}$ is given by:

$X=r \cos (\alpha)+\sqrt{L^{2}-(r \sin (\alpha)+A)^{2}}$

The maximum ' $X_{\max }$ ' position of the piston relative to the axis of the crankshaft is illustrated in the simplified diagram below:

$X_{\text {max }}=\sqrt{(r+L)^{2}-A^{2}}$

We implement the mathematical equation reflecting the functional condition in the 20-sim software [9]. The program translating the mathematical model is:

\section{parameters}

Note that the equation reflecting the functional condition $\left(\mathrm{X}_{\mathrm{f}}=\mathrm{X}_{\max }\right)$ is not linear.

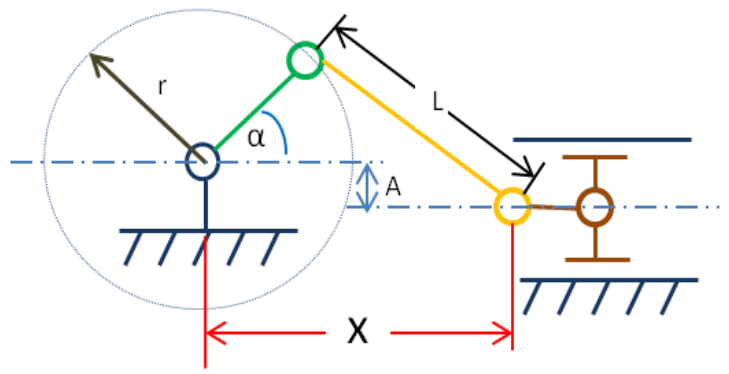

Fig. 5. slider crank system

B. Tolerances analysis "worst case"

The simulation is launched using the extreme values and then we recover ' $\mathrm{L}_{\mathrm{i}}$ ' limits (lower limit) and ' $\mathrm{L}_{\mathrm{s}}$ ' (upper limit) of the functional condition, Then the tolerance interval at "worst case"(IT $\left.\mathrm{wc}_{\mathrm{wc}}\right)$ is deduced. 
TABLE I. PARAMETERS AND NOMINAL DISPERSION

\begin{tabular}{|c|c|c|c|c|c|}
\hline Parameters & Nominal values & Li & Ls & IT & $\boldsymbol{\sigma}$ \\
\hline L & 178 & 177,300 & 178,700 & $\pm 0,70$ & 0,233 \\
\hline r & 39 & 38,300 & 39,700 & $\pm 0,70$ & 0,233 \\
\hline A & 13 & 12,300 & 13,700 & $\pm 0,70$ & 0,233 \\
\hline
\end{tabular}

TABLE II. RESUlts OF DIMENSIONAL TOLERANCING AT WORST CASE

\begin{tabular}{|c|c|c|c|c|}
\hline Parameters & Nominal values & $\mathbf{L}_{\mathbf{i}}$ & $\mathbf{L}_{\mathbf{s}}$ & $\mathbf{I T}_{\mathbf{w c}}$ \\
\hline $\mathrm{L}$ & 178 & 177,3 & 178,7 & $\pm 0,7$ \\
\hline $\mathrm{r}$ & 39 & 38,3 & 39,7 & $\pm 0,7$ \\
\hline $\mathrm{A}$ & 13 & 12,3 & 13,7 & $\pm 0,7$ \\
\hline $\mathrm{C}_{\mathrm{f}}$ & 216,6103 & 215,1642861 & 218,053365 & $\pm 1, \mathbf{4 4 4 5 3 9}$ \\
\hline
\end{tabular}

It is noted from the results of the simulation that:

$$
\begin{aligned}
& \operatorname{Li}\left(\mathbf{C}_{\mathbf{f}}\right)=\sqrt{(\operatorname{Li}(\mathbf{L})+\operatorname{Li}(\mathbf{r}))^{2}-(\operatorname{Ls}(\mathbf{A}))^{2}} \\
& \operatorname{Ls}\left(\mathbf{C}_{\mathbf{f}}\right)=\sqrt{(\operatorname{Ls}(\mathbf{L})+\operatorname{Ls}(\mathbf{r}))^{2}-(\operatorname{Li}(\mathbf{A}))^{2}}
\end{aligned}
$$

The presented method has made it possible to conduct an analysis of the tolerances at "worst case" where the relationship between the resulting functional requirement and basic characteristics is nonlinear. These results guarantee the mechanism operation with parameters within their limits configurations.

\section{Tolerance Analysis by Monte Carlo: statistical approach}

The result of the analysis with Monte Carlo simulation depends on the sample size ' $N$ '. The number of draws ' $N$ ' has to be high in order to get accurate results. Indeed, the accuracy of this statistical analysis increases as a proportion to ' $\sqrt{ } \mathrm{N}$ ' [10].

If the value of $\mathrm{N}$ is sufficiently large, the result of the method reaches a stable value, substantially independent of $\mathrm{N}$. In fact, if the difference between two results of simulation in different draws is greater than $5 \%$, then the number of draws $\mathrm{N}$ is not sufficient to reach stability [11].

These tests determine the extent of the result variations and calculate its standard deviation (Figure 6). We will use a sample size of $\mathrm{N}=10000$ to predict statistically the form of ' $\mathrm{X}_{\max }$ '.

The implementation of the MCS method is to first generate the histograms of Figure 8 based on the values of the table II. We chose tolerance intervals (IT) of \pm 0.70 and homogeneous distribution of $\pm 3 \sigma$ for each parameter.

The histograms in figure 8 show the variation of each parameter and that of ' $\mathrm{C}_{\mathrm{f}}$ ' in their tolerance range. They essentially provide information on the behavior of each parameter within its variation area.

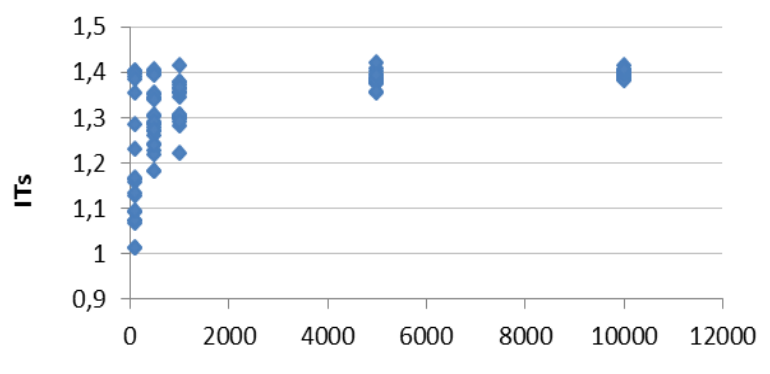

N

Fig. 6. Evolution of the estimated values of tolerance intervals versus "N"

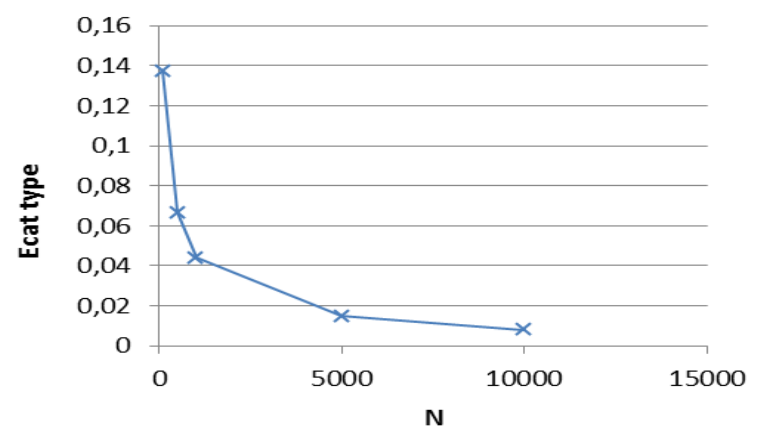

Fig. 7. Evolution of the standard deviation of the estimated tolerance intervals versus "N"

The result of the MCS method is also a random variable. To characterize the random variable, twenty Monte Carlo simulations are launched for each value of "N" (figure8).

The effects of changes in basic parameters on the kinematic behavior of the system are presented in Figure 9.
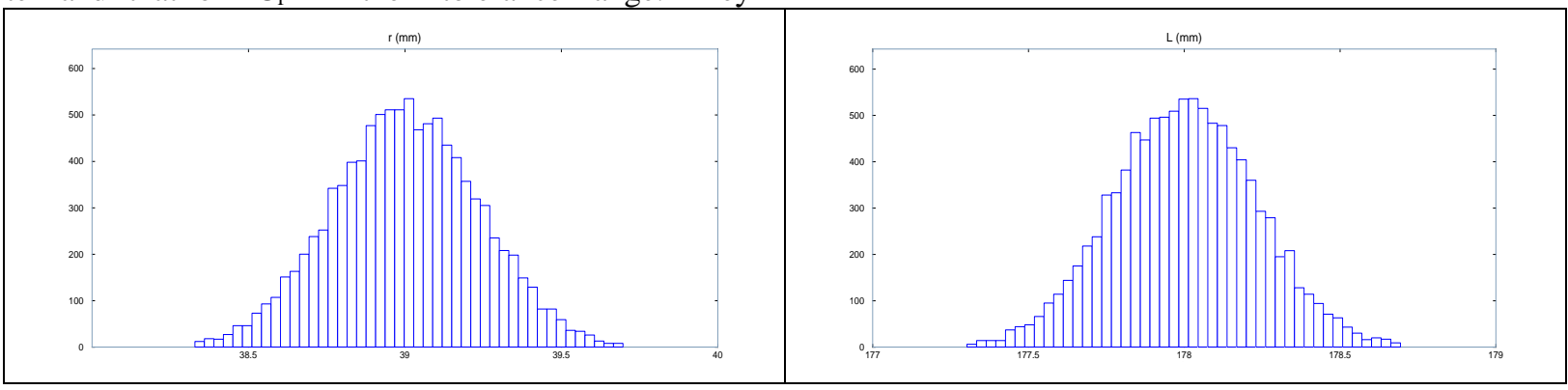


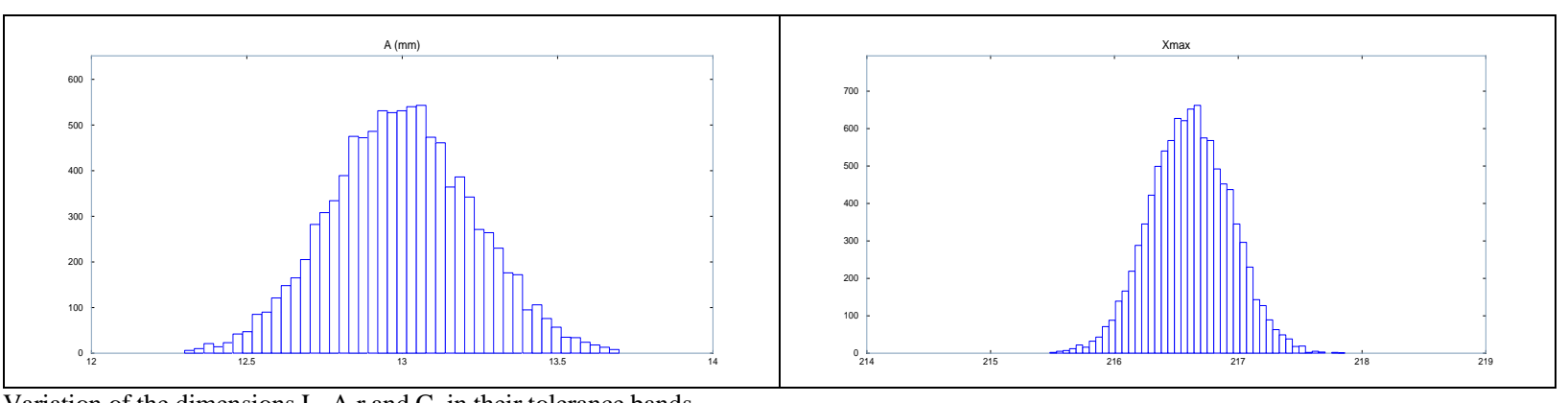

Fig. 8. Variation of the dimensions $\mathrm{L}, \mathrm{A}, \mathrm{r}$ and $\mathrm{C}_{\mathrm{f}}$ in their tolerance bands

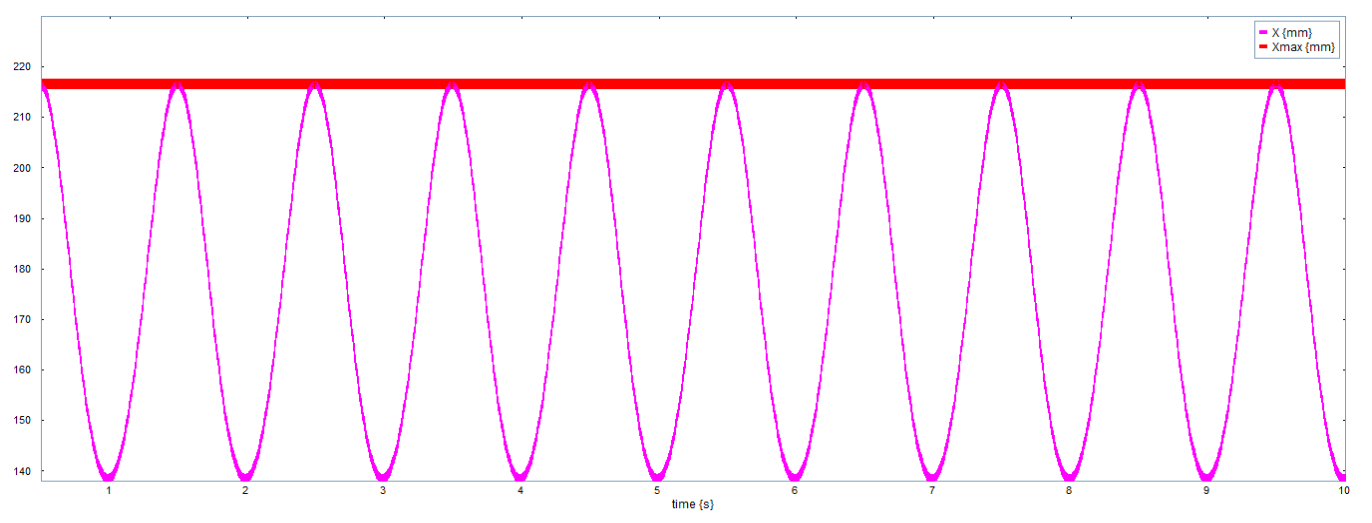

Fig. 9. The effects of changes in parameters $\mathrm{X}$ and $\mathrm{X}_{\max }$

TABLE III. RESULTS OF DIMENSIONAL TOLERANCING WITH MCS

\begin{tabular}{|c|c|c|c|c|c|}
\hline Parameters & Nominal values & $\mathbf{L}_{\mathbf{i}}$ & $\mathbf{L}_{\mathbf{s}}$ & $\mathbf{I T}_{\mathbf{M C S}}$ & $\boldsymbol{\sigma}$ \\
\hline $\mathrm{L}$ & 178 & 177,300 & 178,700 & $\pm 0,70$ & 0,233 \\
\hline $\mathrm{R}$ & 39 & 38,300 & 39,700 & $\pm 0,70$ & 0,233 \\
\hline $\mathrm{A}$ & 13 & 12,300 & 13,700 & $\pm 0,70$ & 0,233 \\
\hline $\mathrm{X}_{\max }$ & 216,610249 & 215,41491 & 217,75478 & $\pm \mathbf{1 , 1 6 9 9 3}$ & 0,390 \\
\hline
\end{tabular}

By comparing the results obtained by the worst case method to those found with MCS, (Table IV), we find that the statistical variation zone is within the area determined by the arithmetic method WC $\left(\mathrm{IT}_{\mathrm{MCS}}<\mathrm{IT}_{\mathrm{WC}}\right)$.

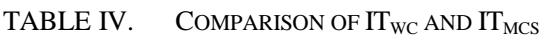

\begin{tabular}{|c|c|c|c|}
\hline $\mathrm{C}_{\boldsymbol{f}}$ & $\mathbf{L}_{\mathbf{i}}(\mathbf{C f})$ & $\mathbf{L}_{\mathbf{s}}(\mathbf{C f})$ & $\mathbf{I T}$ \\
\hline $\mathrm{IT}_{\text {_wC }}$ & 215,1643 & 218,0534 & $\pm 1,4445$ \\
\hline IT_MCS & 215,4149 & 217,7548 & $\pm 1,1699$ \\
\hline
\end{tabular}

\section{Tolerances optimization}

Since the statistical variation area is included in the arithmetic area, the optimization of parameters IT $_{\text {MCS }}$ intervals is possible. For this, we proceed to the expansion of each parameter tolerances intervals, until the new value of the interval $\mathrm{IT}_{\mathrm{MCS}}$ is acceptable ( $\mathrm{IT}_{\mathrm{MCS}}<\mathrm{IT}_{\mathrm{wc}}$ ). The optimization cycle is stopped. For the studied mechanism, the optimization cycle yielded the values shown in the table $\mathrm{V}$.

\section{TABLE V. IT'S OPTIMIZED WITH MCS}

\begin{tabular}{|c|c|c|c|c|c|}
\hline Parameters & Nominal values & $\mathbf{L}_{\mathbf{i}}$ & $\mathbf{L}_{\mathrm{s}}$ & $\mathbf{I T}_{\text {opt }}$ & $\sigma$ \\
\hline $\mathrm{L}$ & 178 & 177,15 & 178,85 & $\pm 0,85$ & 0,283 \\
\hline $\mathrm{r}$ & 39 & 38,15 & 39,85 & $\pm 0,85$ & 0,283 \\
\hline $\mathrm{A}$ & 13 & 12,15 & 13,85 & $\pm 0,85$ & 0,283 \\
\hline $\mathrm{C}_{\mathrm{f} \text { _opti }}$ & 216,610249 & 215,11767 & 217,978238 & $\pm 1,43028$ & 0,477 \\
\hline
\end{tabular}

\section{CONCLUSION}

In this work, a new method based on Monte Carlo simulation and the Worst Case was proposed to analyze nonlinear condition tolerance interval. Tolerance intervals were expanded for different dimensions without affecting the whole system functionality. This will reduce manufacturing costs while maintaining the overall system functionality.
[1] F. Charpentier, " Maîtrise du processus de modélisation géométrique et physique en conception mécanique," Thesis, University of Bordeaux, 2014.

[2] P. A. Adragana, «tolérancement des systèmes assemblés, une approche par le tolérancement inertiel et modal », Thesis, University of Savoie, 2007.

[3] V. Moradinaftchali, L. Song, X. Wang "Improvement in quality and productivity of an assembled product: A riskless approach", Computers and Industrial Engineering, ELSEVIER, Vol. 94, pp. 74-82, April 2016. 
[4] J. U. Turner, "Tolerances in Computer-Aided Geometric Design," Thesis, Faculty of Rensselaer Polytechnique Institute, 1987.

[5] L. Joskowicz, E. Sacks,V. Srinivasan, " Kinematic tolerance analysis.," Computer-Aided Design, vol. 29, pp. 147-157, 1997.

[6] Y. Ostrovsky-berman,L. Joskowicz, "Tolerance envelopes of planar mechanical parts with parametric tolerances," Computer-Aided Design, vol. 37, pp. 531-544, 2005.

[7] M. Temmreman, "Analyse et synthèse au pire des cas et statistique dans l'environnement CFAO," Thèse de doctorat, LISMMA-ISMACM, Ecole Centrale de Paris, Paris, 2001.
[8] J. U. Turner, " A feasability space approach for automated tolerancing," Journal of Engineering for industry, pp. 341-346, 1993.

[9] 20-sim ${ }^{\circledR}$ home page: http://www.20sim.com/product/bondgraphs.html

[10] R. CVETKO, CHASE, K. W., MALEBY, S. P., "New metrics for evaluating Monte Carlo tolerance analysis of assemblies," Proceedings of the ASME International Mechanical Engineering Conference and Exposition, 1998

[11] N. JOUILEL, M. RADOUANI, M. EL GADARI, B. EL FAHIME, "Mechatronic tolerancing: Bond Graph approach". COMPUSOFT, An International Journal of Advanced Computer Technology, Vol. 5, Issue no 2, pp. 2063-2070, February 2016. 